\title{
Potential Role of Dexamphetamine in the Treatment of Non-alcoholic Fatty Liver Disease: Hopes and Pitfalls
}

\author{
CS Gautam, ${ }^{1}$ Jatin Sharma, ${ }^{2}$ Mandeep Singla, ${ }^{1}$ IImjot Kaur Tiwana ${ }^{3}$ and Harmanjit Singh ${ }^{1}$ \\ 1. Department of Pharmacology, Government Medical College and Hospital, Chandigarh, India; 2. Department of Pharmacology, All India \\ Institute of Medical Sciences, New Delhi, India; 3. Government Medical College and Rajindra Hospital, Patiala, India
}

DOI: https://doi.org/10.17925/EE.2021.17.1.33

$\mathrm{N}$ on-alcoholic fatty liver disease (NAFLD) is one of most frequent causes of chronic liver disease. Global prevalence of NAFLD and nonalcoholic steatohepatitis (NASH) with advanced fibrosis is increasing day by day. Patients with NAFLD are more susceptible to encounter cardiovascular morbidity and mortality. Apart from lifestyle changes and dietary modifications, no effective pharmacotherapy is available to prevent the progression of NAFLD to NASH and advanced stages of hepatic fibrosis and cirrhosis. Dexamphetamine is the d-isomer of amphetamine, which acts by inhibiting monoamine reuptake and direct stimulation of dopamine and noradrenaline release. Presently, dexamphetamine is indicated for the treatment of attention deficit hyperactivity disorder and narcolepsy, but since its use was found to be associated with weight loss, it is also now used as an off-label drug for the treatment of obesity. Direct or indirect evidence is present in the form case reports, case series and from effects of related drugs to support the potential role of dexamphetamine in NAFLD. There is an urgent need to initiate preclinical and clinical studies involving robust methodology and adequate sample sizes to explore the potential of dexamphetamine in patients with NAFLD. In this review, we will discuss the therapeutic potential of dexamphetamine for the treatment of NAFLD.

\section{Keywords}

Chronic liver disease, non-alcoholic steatohepatitis, cirrhosis, hepatic steatosis

Disclosures: CS Gautam, Jatin Sharma, Mandeep Singla, IImjot Kaur Tiwana and Harmanjit Singh have no financial or non-financial relationships or activities to declare in relation to this article. Review process: Double-blind peer review.

Compliance with ethics: This study involves a review of the literature and did not involve any studies with human or animal subjects performed by any of the authors. Authorship: The named authors meet the International Committee of Medical Journal Editors (ICMJE) criteria for authorship of this manuscript, take responsibility for the integrity of the work as a whole, and have given final approval for the version to be published.

Access: This article is freely accessible at

touchENDOCRINOLOGY.com ( ) Touch Medical Media 2021.

Received: 25 June 2020

Accepted: 14 January 2021

Published online: 31 March 2021

Citation: touchREVIEWS in Endocrinology.

2021;17(1):33-6

Corresponding author: Harmanjit Singh,

Department of Pharmacology Room E-509, 5th

Level, E-Block, Govt. Medical College and Hospital,

Chandigarh-160030 India. E: harman_gmcp@yahoo.com

Support: No funding was received for

the publication of this article.
Non-alcoholic fatty liver disease (NAFLD) is the most frequent cause of chronic liver disease globally; it is histologically classified into non-alcoholic fatty liver (NAFL) and non-alcoholic steatohepatitis (NASH). ${ }^{1}$ NAFL is characterized by the presence of $>5 \%$ of hepatic steatosis without evidence of hepatocellular injury in the form of hepatocyte ballooning. NASH is the advanced stage of NAFLD comprising $>5 \%$ of hepatic steatosis and inflammation, along with hepatocyte injury. ${ }^{2}$ If not treated in the early stage, NAFL can progress to NASH, hepatic fibrosis and cirrhosis. It has been recognized as the most frequent indication for hepatic transplantation. ${ }^{3}$ Furthermore, it is one of the leading causes of hepatocellular carcinoma. ${ }^{4}$

The risk of NAFLD increases with the presence of any component of metabolic syndrome, such as obesity, hypertension, high cholesterol and type 2 diabetes mellitus. ${ }^{5}$ Considering the importance of metabolic derangements and their impact on the pathogenesis of NAFLD, new nomenclature has been proposed for this disease entity: metabolic associated fatty liver disease (MAFLD). ${ }^{6,7}$ The need to rename NAFLD to MAFLD was highlighted by a group of researchers (comprised of an international consensus panel) from the Westmead Institute for Medical Research since they felt that the new terminology will define its aetiology in a clearer way and improve public health initiatives.? Hence, the term NAFLD is likely to be replaced by MAFLD. The worldwide prevalence of NAFLD is estimated to be $25 \%{ }^{8}$ In western countries, overall prevalence of NAFLD is 15-40\%, whereas epidemiological studies suggest the prevalence in the Indian population to be around 9-32\%, with a greater incidence in patients suffering from obesity and diabetes. ${ }^{9}$

Epidemiological studies have shown an increased mortality with advanced fibrosis, which demands the urgent requirement of effective pharmacotherapy in patients with NAFLD. ${ }^{9}$ Considering the epidemic increase in the prevalence of NAFLD, its associated morbidity and mortality, and its rising burden of healthcare, there is a dire need to develop effective pharmacological approaches. This review will focus on the potential role of dexamphetamine in the treatment of NAFLD.

\section{Current approaches for management of non-alcoholic fatty liver disease}

There are no approved pharmacotherapeutic options currently available for the treatment of NAFLD. ${ }^{10}$ However, currently available treatment strategies for NAFLD consist of weight reduction, lifestyle modifications and treatment of the components of metabolic syndrome. ${ }^{10}$ In various randomized controlled trials, weight reduction has shown to improve liver enzymes, histology 
and insulin levels in patients with NAFLD, and it has been suggested that weight loss is the key factor to improve liver histology in patients suffering from NASH. ${ }^{11-13}$ There is some supporting evidence regarding the use of insulin sensitizers, like pioglitazone and vitamin E; however, their use is not considered in all patients. ${ }^{14}$ Pharmacological treatment is typically reserved for patients who have failed to reach their weight-loss goal and those with biopsy-proven NASH with fibrosis stage $\geq 2 .{ }^{13}$ Trials addressing pharmacologic therapies have not been large or particularly informative enough to determine the impact of these therapies on important clinical outcomes, such as decompensated cirrhosis. ${ }^{13}$ Most of these trials addressed surrogate outcomes, such as serum aminotransferase levels or liver histology, and too often with conflicting results. ${ }^{13}$

In the guidelines provided by the American Association for the Study of Liver Disease and European Association for the Study of the Liver, a daily dose of 800 international units of vitamin $E$ has been recommended for patients with NASH (biopsy-proven) without diabetes mellitus. ${ }^{2}$ Vitamin $\mathrm{E}$ has been shown to improve steatosis and inflammation, but it has not shown any effect on improvement of hepatic steatosis. ${ }^{2}$ However, a meta-analysis by Musso et al. revealed that there are no histologic benefits with the administration of vitamin E. ${ }^{13}$ However, this meta-analysis suffered from significant heterogeneity in terms of the formulations of vitamin E used, the patient participants, treatment duration and lifestyle modifications. ${ }^{13}$ Pioglitazone is also recommended for the treatment of patients with histology-proven NASH with or without diabetes mellitus, but its use is largely restricted because of its potential adverse effects..$^{15} \mathrm{~A}$ meta-analysis by Li et al. in patients with NAFLD showed that metformin can improve body mass index (BMI), insulin resistance and hepatic transaminase levels, but does not improve steatosis and liver histology. ${ }^{16}$

Farnesoid $X$ nuclear receptor (FXR) ligand, obeticholic acid, is being investigated for its potential role in the management of NAFLD. ${ }^{17}$ FXR has a prominent role in the synthesis and enterohepatic circulation of bile acids, and also regulates the lipoprotein, immune inflammatory and fibrotic pathways important in NAFLD. Obeticholic acid acts as an FXR agonist and it helps in correcting the abnormalities in cholesterol lipoprotein and bile acid metabolism, and modulates the immuno-inflammatory and fibrogenic changes seen in NAFLD. Obeticholic acid has shown promising results in clinical trials and has led to improvements in liver histology, insulin sensitivity, and has reduced markers of liver inflammation and fibrosis. ${ }^{18,19}$

\section{Dexamphetamine in non-alcoholic fatty liver disease: potential role and current evidence}

Dexamphetamine is the d-isomer of amphetamine, which is an indirectly-acting sympathomimetic drug, inhibiting reuptake of monoamines and direct stimulation of release of dopamine and noradrenaline. ${ }^{20}$ Dexamphetamine firstly enters the presynaptic neuron where it binds to the vesicular monoamine transporter-2. It prevents the translocation of monoamine from the cytosolic pool to storage vesicles, leading to intracellular monoamine increases. This also stimulates the amine-associated receptor intracellular trace1 to promote dopamine efflux. All these processes help to reverse the transportation through noradrenaline or dopamine transporter, enhancing extracellular monoamine release. Elevated release of monoamines promotes satiety and reduces feeding through the activation of postsynaptic $\alpha$ - and $\beta$-adrenergic receptors and D1/D2 receptors. ${ }^{21}$ The $d$-isomer of amphetamine is more potent than its 1 -isomer. ${ }^{22}$ It has an extensive distribution in most of the tissues and possesses a potent psycho-stimulant effect. After oral administration, a high concentration is achieved in the central nervous system, and it produces various effects, such as suppression of appetite and an improvement in concentration and cognitive functions. ${ }^{23}$ Currently, it is indicated for the treatment of attention deficit hyperactivity disorder and narcolepsy. Use of dexamphetamine was found to be associated with weight loss; hence, it is used as an off-label drug for the treatment of obesity. ${ }^{24,25}$

A case series published by Ghazala et al. revealed promising outcomes in two patients with Dercum's disease. ${ }^{26}$ In case 1 , in a 55-year-old man, administration of dexamphetamine for 3 months led to a reduction in weight and hepatic steatosis. Initially dexamphetamine was started at a dose of $10 \mathrm{mg}$ but then reduced to $7.5 \mathrm{mg}$ due to anxiety and jitteriness. Similar results were observed in case 2, a 52-year-old female with Dercum's disease. The drug was initially started at a dose of 1.25 $\mathrm{mg}$ daily and was gradually increased to $20 \mathrm{mg}$ daily. A year later magnetic resonance imaging also showed an improvement in hepatic steatosis. ${ }^{26}$ Although the case series does not carry a significant weight towards evidence-based medicine, the promising results obtained from this particular case series demand further clinical studies involving adequate sample size.

A retrospective study by Ismail et al. in patients with structural hypothalamic lesions revealed that dexamphetamine $5 \mathrm{mg}$ twice daily for 15 months led to weight loss and improvement in exercise tolerance. ${ }^{27}$ Another retrospective case series study by Denzer et al. in seven patients suffering from hypothalamic obesity also led to promising results..$^{28}$ In all patients, dexamphetamine was started at $5 \mathrm{mg}$ per day and gradually increased to up to $20 \mathrm{mg}$ per day. In all patients with hypothalamic obesity, a reduction in BMI was observed compared to baseline and no troublesome adverse effects were observed. ${ }^{28}$

Mason et al. performed a prospective study on five children who had a poor attention and history of significant weight gain following surgical treatment for craniopharyngioma. ${ }^{28}$ In this study, treatment with dexamphetamine was started at $5 \mathrm{mg}$ and was increased gradually by $2.5 \mathrm{mg}$ per week until adverse effects and decrease in appetite were observed. Dexamphetamine also stabilized the weight gain and reduced BMI from $32 \pm 2.8$ at the start of treatment to $31 \pm 3.3$ after 24 months of study. Administration of dexamphetamine also showed an improvement in overall activity and attention of children included in this study. ${ }^{28}$

In a pilot study by Poulton et al., the anorexigenic effect of dexamphetamine was evaluated for promoting weight loss.30 Obese adults were treated with dexamphetamine for 6 months at a maximum dose of $30 \mathrm{mg}$ day in addition to diet and exercise. This approach led to a significant weight reduction of $10.6 \mathrm{~kg}(\mathrm{p}<0.001)$. After stopping the therapy with dexamphetamine there was a rebound gain in weight of 4.5 $\mathrm{kg}$ (in 6 months $\mathrm{p}=0.003$ ). In addition, no cardiac adverse events were observed. Hence, this study provided important preliminary evidence that dexamphetamine in addition to diet and exercise may offer a safe and effective approach for weight loss, and this may also be helpful in patients with NAFLD. Evidence from clinical studies has been summarized in Table 1.

\section{Indirect evidence}

Although this section does not address the role of dexamphetamine per se, we have narrated the evidence obtained from related compounds with similar mechanisms to that of dexamphetamine. In a preclinical study by Decara et al. on obese Zucker rats, it was found that treatment with oleic acid and dihydroxyamphetamine, given for 15 days, reduced histologicallyconfirmed hepatic steatosis as well as plasma triglyceride levels. ${ }^{31}$ 
Table 1: Evidence from clinical studies regarding potential use of dexamphetamine in non-alcoholic fatty liver disease

\begin{tabular}{|c|c|c|}
\hline Study & Study design & Findings \\
\hline Ghazala et al., $2018^{25}$ & Case series & Dexamphetamine significantly reduced hepatic steatosis in patient suffering from Dercum's disease \\
\hline Ismail et al., $2006^{26}$ & $\begin{array}{l}\text { Retrospective review of } \\
\text { case notes }\end{array}$ & $\begin{array}{l}\text { Dexamphetamine at a dose of } 5 \mathrm{mg} \text { twice daily for } 15 \text { months caused weight loss and improved } \\
\text { exercise tolerance in patients with structural hypothalamic lesions }\end{array}$ \\
\hline Denzer et al., $2019^{27}$ & Retrospective case series & In patients with hypothalamic obesity, dexamphetamine administration significantly reduced BMI \\
\hline Mason et al., $2002^{28}$ & Prospective clinical study & $\begin{array}{l}\text { Treatment with dexamphetamine led to stabilization in weight gain and reduced BMI from } 32 \pm 2.8 \text { at } \\
\text { the start of treatment to } 31 \pm 3.3 \text { after } 24 \text { months of study. } \\
\text { This also led to improvement in overall activity and attention of children }\end{array}$ \\
\hline Poulton et al., $2015^{29}$ & Open-label pilot study & $\begin{array}{l}\text { Obese adults who were treated with dexamphetamine (for } 6 \text { months) at a maximum dose of } \\
30 \mathrm{mg} / \mathrm{day} \text { (in addition to diet, and exercise) showed a significant weight reduction by } 10.6 \mathrm{~kg} \\
(\mathrm{p}<0.001) \text {. After stopping therapy with dexamphetamine there was a rebound gain in weight by } \\
4.5 \mathrm{~kg} \text { (in } 6 \text { months } p=0.003 \text { ). No cardiac adverse effects were observed }\end{array}$ \\
\hline
\end{tabular}

$B M I=$ body mass index.

Sibutramine (a serotonin and norepinephrine reuptake inhibitor that acts by increasing energy expenditure and satiety), an older anti-obesity drug, has been shown to be effective in the treatment of NAFLD. In a study by Sabuncu et al. on obese patients suffering from NASH with mean BMl of $37.3 \mathrm{~kg} / \mathrm{m}^{2}$, it was found that treatment with sibutramine for 6 months significantly reduced body weight, hepatic steatosis and insulin resistance. Improvement in levels of serum transaminases was also observed, except for alkaline phosphate. ${ }^{31}$ One randomized, double blind, placebo-controlled trial in 92 participants has been planned to evaluate the safety and tolerability of phentermine $15 \mathrm{mg}$ for 6 weeks for the reduction of hepatic steatosis, adipose tissue and postoperative complications in patients undergoing bariatric surgery and those requiring bariatric surgery. ${ }^{33}$

\section{Potential safety concerns of dexamphetamine}

Dexamphetamine is well tolerated and is rarely associated with troublesome adverse events. Neurological adverse effects are mainly observed in patients suffering from previous psychiatric illnesses. ${ }^{34}$ Neuropsychiatric adverse effects caused by dexamphetamine include insomnia, anxiety, confusion and delusions. Other adverse effects include gastrointestinal events (nausea, abdominal discomfort) and retention of urine. Hypertension and cardiac arrhythmias can occur at higher doses, but they tend to resolve after dose reduction..$^{21,35} \mathrm{~A}$ study by Williams et al. showed that dexamphetamine has been a less commonly abused drug compared to abuse of other substances. ${ }^{35}$ Apart from this, dexamphetamine can lead to some clinically significant drug interactions with drugs such monoamine oxidase inhibitors; antacids and other drugs used for peptic ulcers; antipsychotics, such as chlorpromazine and haloperidol; norepinephrine; and anticonvulsants, such as phenytoin and phenobarbital. As such, caution should be taken while prescribing dexamphetamine along with potentially interacting drugs. ${ }^{36,37}$

\section{Future considerations}

The prevalence of NAFLD is increasing day by day, and currently no effective pharmacotherapeutic options are available to address this issue. ${ }^{9}$ Published literature has shown the promising effects of dexamphetamine in hypothalamic obesity and NAFLD. ${ }^{26-30}$ Although dexamphetamine has some neuropsychiatric, gastrointestinal and cardiovascular adverse effects, ${ }^{21,34,35}$ the potential benefits may outweigh the risks. Introduction of the term MAFLD in place of NAFLD reflects the importance of metabolic parameters in the pathogenesis of this clinical entity. ${ }^{6,7} \mathrm{As} \mathrm{BMI}$ and weight, along with factors such as insulin resistance, play a crucial role in the development of MAFLD, 5,6,7 dexamphetamine can play a crucial role in addressing these metabolic derangements. Studies assessing the preclinical effect of dexamphetamine in NAFLD are lacking; therefore, more preclinical studies are required to explore the potential effect of dexamphetamine for the treatment of NAFLD. In addition, there is a dire need for clinical studies involving robust methodology and adequate sample size to explore the potential of dexamphetamine in patients with NAFLD. This can also help in defining the dose range of dexamphetamine for this indication. However, from the available evidence, it can be said that dexamphetamine could emerge as a potential therapeutic option for the treatment of NAFLD.

\section{Conclusion}

Dexamphetamine appears to possess promising potential for the treatment of NAFLD. However, it has some safety concerns and further data are needed on its efficacy. To fulfil the unmet need of NAFLD treatment, there is a significant need to undertake research and generate a high level of evidence to ascertain its efficacy and safety for the treatment of NAFLD. $\square$
1. Chalasani $\mathrm{N}$, Younossi Z, Lavine JE, et al. The diagnosis and management of non-alcoholic fatty liver disease: practice guideline by the American Gastroenterological Association, guideline by the American Gastroenterological Association, American Association for the Study of Liver Diseases, and American College of Gastroenterology. Gastroenterology. 2012;142:1592-609.

2. Chalasani $\mathrm{N}$, Younossi Z, Lavine JE, et al. The diagnosis and management of nonalcoholic fatty liver disease: Practice guidance from the American Association for the Study of Liver Diseases. Hepatology. 2018;67:328-57.

3. Charlton MR, Burns JM, Pedersen RA, et al. Frequency and outcomes of liver transplantation for nonalcoholic steatohepatitis in the United States. Gastroenterology. 2011;141:1249-53

4. Duseja A. Nonalcoholic fatty liver disease in India - a lot done, yet more required! Indian J Gastroenterol. 2010;29:217-25.

5. Younossi ZM, Koenig AB, Abdelatif D, et al. Global epidemiology of nonalcoholic fatty liver disease- Meta-analytic assessment of prevalence, incidence and outcomes. Hepatology.

\section{6:64:73-84}

6. Eslam M Sanyal AL George J. MAFLD: a consensus-driven proposed nomenclature for metabolic associated fatty liver disease. Gastroenterology. 2020;158:1999-2014.

7. Medical Xpress. MAFLD: A new name and definition brings hope for patients with fatty liver disease. Available at: https://medicalxpress.com/news/2020-03-mafld-definitionpatients-fatty-liver.html (accessed 28 January 2021).

8. Kalra S, Vithalani M, Gulati G, et al. Study of prevalence of nonalcoholic fatty liver disease (NAFLD) in type 2 diabetes patients in India (SPRINT). J Assoc Physicians India. 2013;61:448-53

9. Dulai PS, Singh S, Patel J, et al. Increased risk of mortality by fibrosis stage in nonalcoholic fatty liver disease: systematic review and meta-analysis. Hepatology. 2017;65:1557-65.

10. Geier S, Rau M. Emerging therapies for NASH - the future is now. Expert Rev Clin Pharmacol. 2017;10:467-9.

11. Dixon JB, Bhathal PS, Hughes NR, O'Brien PE. Nonalcoholic fatty liver disease: Improvement in liver histological analysis with weight loss. Hepatology. 2004:39:1647-54

12. Bhat G, Baba CS, Pandey A, et al. Life style modification improves insulin resistance and liver histology in patient with non-alcoholic fatty liver disease. World I Hepato. 2012;4:209-17.

13. Musso G, Gambino R, Cassader M, Pagano G. A meta-analysis of randomized trials for the treatment of nonalcoholic fatty live disease. Hepatology. 2010;52:79-104.

14. Tilg $H$, Moschen AR, Roden M. NAFLD and diabetes mellitus. Nat Rev Gastroenterol Hepatol. 2017;14:32-42.

15. Cusi K, Orsak B, Bril F, et al. Long-term pioglitazone treatment for patients with nonalcoholic steatohepatitis and prediabetes or type 2 diabetes mellitus: a randomized trial. Ann Intern Med. 2016;165:305-15.

16. Li Y, Liu L, Wang B, et al. Metformin in non-alcoholic fatty liver disease: A systematic review and meta-analysis. Biomed Rep. 2013:1:57-64.

17. Makri E, Cholongitas E, Tziomalos K. Emerging role of obeticholic acid in the management of nonalcoholic fatty liver 


\section{Review Liver Disorders}

disease. World I Gastroenterol. 2016;22:9039-43.

18. Neuschwander-Tetri BA, Loomba R, Sanyal AJ, et al. Farnesoid X nuclear receptor ligand obeticholic acid for non-cirrhotic, non-alcoholic steatohepatitis (FLINT): a multicentre, randomised, placebo-controlled trial. Lancet 2015:385:956-65. [Erratum in 'Lancet. 2015:385:946. Erratum in Lancet. 2016;387:1618.]

19. Mudaliar S, Henry RR, Sanyal AJ, et al. Efficacy and safety of the farnesoid $\mathrm{X}$ receptor agonist obeticholic acid in patients with type 2 diabetes and nonalcoholic fatty liver disease. Gastroenterology. 2013;145:574-82.

20. Rizzo R, Gulisano M. Clinical pharmacology of comorbid attention deficit hyperactivity disorder in Tourette syndrome. Int Rev Neurobiol. 2013;112:415-44.

21. Stemmer K, Müller TD, DiMarchi RD, et al. CNS-targeting pharmacological interventions for the metabolic syndrome. J Clin Invest. 2019;129:4058-71.

22. Heal DJ, Smith SL, Gosden J, Nutt DJ. Amphetamine, past and present a pharmacological and clinical perspective. J Psychopharmacol. 2013:27:479-96.

23. Angrist B, Corwin J, Bartlik B, Cooper T. Early pharmacokinetics Angrist B, Corwin J, Bartlik B, Cooper T. Early pharmacokinetics
and clinical effects of oral D-amphetamine in normal subjects. and clinical effects of oral D-amphet
Biol Psychiatry. 1987;22:1357-68.

24. Haddock CK, Poston WS, Dill PL, et al. Pharmacotherapy form obesity: a quantitative analysis of four decades of published randomized clinical trials. Int I Obes Relat Metab Disord. 2002:26:262-73.

25. Schrantee A, Tremoleda JL, Wylezinska-Arridge $\mathrm{M}$, et a Repeated dexamphetamine treatment alters the dopaminergic system and increases the phMRI response to methylphenidate. PLOS One. 2017;12:e0172776.

26. Ghazala S, Bilal J, Ross E, et al. Low-dose d-amphetamine induced regression of liver fat deposits in Dercum disease. Am J Med. 2018;131:705-8.

27. Smail D, O'Connell MA, Zacharin MR. Dexamphetamine use for management of obesity and hypersomnolence following hypothalamic injury. J Pediatr Endocrinol Metab. 2006;19:129-34.

28. Denzer C, Denzer F, Lennerz BS, et al. Treatment of hypothalamic obesity with dextroamphetamine: a case series Obes Facts. 2019:12:91-102.

29. Mason PW, Krawiecki N, Meacham LR. The use of dextroamphetamine to treat obesity and hyperphagia in children treated for craniopharyngioma. Arch Pediatr Adolesc Med. 2002;156:887-92.

30. Poulton AS, Hibbert EJ, Champion BL, et al. Piloting a new approach to the treatment of obesity using dexamphetamine. Front Endocrinol (Lausanne). 2015;6:14.

31. Decara JM, Pavón FJ, Suárez J, et al. Treatment with a novel oleic-acid dihydroxyamphetamine conjugation ameliorates non-alcoholic fatty liver disease in obese Zucker rats. Dis Mode Mech. 2015;8:1213-25.

32. Sabuncu T, Nazligul Y Karaoglanoglu M, et al. The effects of sibutramine and orlistat on the ultrasonographic findings, insulin resistance and liver enzyme levels in obese patients with non-alcoholic steatohepatitis. Rom I Gastroenterol. with non-alcoho

33. ClinicalTrials. gov. Effectiveness and Tolerability of Phentermine in Patients Under Bariatric Surgery. ClinicalTrials.gov Identifier. NCT03849729. Available at: https://clinicaltrials.gov/ct2/show/ NCT03849729 (accessed 28 January 2021).

34. Long D, Young J. Dexamphetamine treatment in stroke. QJM. 2003; $96: 673-85$

35. Williams RJ, Goodale LA, Shay-Fiddler MA, et al. Methylphenidate and dextroamphetamine abuse in substance-abusing adolescents. Am J Addict. 2004;13:381-9. DEXEDRINE ${ }^{\oplus}$ (dextroamphetamine sulfate) SPANSUUE sustained-release capsules and Tablets. Prescribing information. Available at: www.accessdata.fda.gov/drugsatfda docs/label/2007/017078s042lbl.pdf (accessed 28 January 2021).

37. Drugs.com. Amphetamine/dextroamphetamine Drug Interactions. Available at: www.drugs.com/drug-interactions/amphetaminedextroamphetamine.html (accessed 28 January 2021). 